\title{
A DIFFERENTIAL INCLUSION: THE CASE OF AN ISOTROPIC SET*
}

\author{
Gisella $\mathrm{CrOCE}^{1}$
}

Abstract. In this article we are interested in the following problem: to find a map $u: \Omega \rightarrow \mathbb{R}^{2}$ that
satisfies
\[ \begin{cases}D u \in E & \text { a.e. in } \Omega \\ u(x)=\varphi(x) & x \in \partial \Omega\end{cases} \]
where $\Omega$ is an open set of $\mathbb{R}^{2}$ and $E$ is a compact isotropic set of $\mathbb{R}^{2 \times 2}$. We will show an existence theorem under suitable hypotheses on $\varphi$.

Mathematics Subject Classification. 34A60, 35F30, 52A30.

Received May 14, 2004.

\section{INTRODUCTION}

In this article we study the following problem: let $\Omega$ be an open set of $\mathbb{R}^{2}$; we investigate the existence of maps $u: \Omega \rightarrow \mathbb{R}^{2}$ (weakly differentiable) that satisfy

$$
\begin{cases}D u \in E & \text { a.e. in } \Omega \\ u(x)=\varphi(x) & x \in \partial \Omega\end{cases}
$$

where $\varphi: \bar{\Omega} \rightarrow \mathbb{R}^{2}$ is sufficiently regular and $E$ is a compact isotropic set of $\mathbb{R}^{2 \times 2}$ (that is $A E B \subseteq E$ for every $A, B \in \mathcal{O}(2))$. In an equivalent way $E$ can be written as

$$
E=\left\{\xi \in \mathbb{R}^{2 \times 2}:\left(\lambda_{1}(\xi), \lambda_{2}(\xi)\right) \in K\right\}
$$

for some compact set $K \subset T=\left\{(x, y) \in \mathbb{R}^{2}: 0 \leq x \leq y\right\}$, where we have denoted by $\lambda_{1}(\xi) \leq \lambda_{2}(\xi)$ the singular values of the matrix $\xi$, that is the eigenvalues of the matrix $\sqrt{\xi \xi^{t}}$, which are

$$
\begin{aligned}
& \lambda_{1}(\xi)=\frac{1}{2}\left[\sqrt{\|\xi\|^{2}+2|\operatorname{det}(\xi)|}-\sqrt{\|\xi\|^{2}-2|\operatorname{det}(\xi)|}\right] \\
& \lambda_{2}(\xi)=\frac{1}{2}\left[\sqrt{\|\xi\|^{2}+2|\operatorname{det}(\xi)|}+\sqrt{\|\xi\|^{2}-2|\operatorname{det}(\xi)|}\right] .
\end{aligned}
$$

Keywords and phrases. Rank one convex hull, polyconvex hull, differential inclusion, isotropic set.

* Research supported by Fonds National Suisse (21-61390-00).

${ }^{1}$ Département de Mathématiques, EPFL, 1015 Lausanne, Switzerland; e-mail: croce@math.univ-montp2.fr 
We will assume throughout the article that

$$
\min \{x,(x, y) \in K\}>0
$$

Thanks to the properties of the singular values (see [6]), the problem (1.1) can be rewritten in the following equivalent way:

$$
\begin{cases}\|D u(x)\|^{2}=a^{2}+b^{2} & \text { a.e. in } \Omega,(a, b) \in K, \\ |\operatorname{det} D u(x)|=a b & \text { a.e. in } \Omega,(a, b) \in K, \\ u(x)=\varphi(x) & x \in \partial \Omega .\end{cases}
$$

We will show the following existence theorem for the problem (1.1):

Theorem 1.1. Let $E$ be defined by (1.2) where $K \subset T$ is a compact set such that $\min \{x,(x, y) \in K\}>0$. Let $\Omega \subset \mathbb{R}^{2}$ be an open set. Let $\varphi \in C_{\text {piec }}^{1}\left(\bar{\Omega} ; \mathbb{R}^{2}\right)$ such that $D \varphi(x) \in E \cup$ int Rco $E$ in $\Omega$ (where Rco $E$ denotes the rank one convex hull of $E$ and int $\operatorname{Rco} E$ its interior $)$. Then there exists a map $u \in \varphi+W_{0}^{1, \infty}\left(\Omega ; \mathbb{R}^{2}\right)$ such that $D u \in E$ a.e. in $\Omega$.

Our result will be a generalization of an existence theorem by Dacorogna and Marcellini (see [3]), where they investigated the case in which $K$ contains only one point, but $K \subseteq \mathbb{R}^{n}, n \geq 2$.

To establish our theorem we will use an abstract existence result proved by Dacorogna and Marcellini [3] and then refined by Dacorogna and Pisante [4], which is based on a functional analytic method, that uses the Baire category theorem. However we recall that the kind of problem like (1.1) can also be solved by another method, established by Gromov [5] and then presented by Müller and Šverák (see [8] for example) in a more analytic manner.

Moreover it will be useful the representation of the rank one convex hull of the set $E$ (we will show that the rank one convex hull coincides with the polyconvex one): for this, we will apply some results established by Cardaliaguet and Tahraoui in [1].

\section{DEFINITIONS AND PRELIMINARY RESULTS}

This section is devoted to the study of the polyconvex hull of an isotropic compact set of $\mathbb{R}^{2 \times 2}$ : this is useful to study the rank one convex hull, as we will see in the next section.

We first give the definitions of polyconvex hull and rank one convex hull of a set $E \subseteq \mathbb{R}^{2 \times 2}$ : we will follow the definitions of Dacorogna and Marcellini [3].

Definition 2.1. Let $E \subseteq \mathbb{R}^{2 \times 2}$; if $\overline{\mathbb{R}}=\mathbb{R} \cup\{+\infty\}$, we define

$$
\begin{aligned}
& \text { Pco } E=\left\{\xi \in \mathbb{R}^{2 \times 2}: f(\xi) \leq 0, \forall f: \mathbb{R}^{2 \times 2} \rightarrow \overline{\mathbb{R}} \text { polyconvex, }\left.f\right|_{E} \leq 0\right\} \\
& \text { Rco } E=\left\{\xi \in \mathbb{R}^{2 \times 2}: f(\xi) \leq 0, \forall f: \mathbb{R}^{2 \times 2} \rightarrow \overline{\mathbb{R}} \text { rank one convex, }\left.f\right|_{E} \leq 0\right\}
\end{aligned}
$$

\section{Remark 2.2.}

i) We observe that, according to this definition, the rank one convex hull of a compact set is not necessarily closed (some examples can be found in [7]). According to our definition Rco $E$ is the smallest rank one convex set which contains $E$ (see [3]). Some authors call our envelop the laminate convex hull of $E$.

ii) We will use also the following representation for Rco $E$ (see [3]): Rco $E=\bigcup_{i \in \mathbb{N}} \mathrm{R}_{i} \operatorname{co} E$, where

$$
\mathrm{R}_{0} \operatorname{co} E=E, \quad \mathrm{R}_{i+1} \operatorname{co} E=\left\{t A+(1-t) B: A, B \in \mathrm{R}_{i} \operatorname{co} E, r k(A-B) \leq 1, t \in[0,1]\right\} .
$$

iii) If $E$ is bounded, then (see [3])

$$
\overline{\mathrm{Pco} E}=\left\{\xi \in \mathbb{R}^{2 \times 2}: f(\xi) \leq 0, \forall f: \mathbb{R}^{2 \times 2} \rightarrow \mathbb{R} \text { polyconvex, }\left.f\right|_{E} \leq 0\right\}=\operatorname{Pco} \bar{E} \text {. }
$$


This means that for a compact set, our notion of polyconvexity coincides with that one of Cardaliaguet and Tahraoui in [1]. We observe that the polyconvex hull of a compact set $E$ is compact.

iv) We recall that Rco $E \subseteq \operatorname{Pco} E$ for every set $E$. [1]:

We now pass to the study of the polyconvex hull of our set $E$. We recall the following result established in

Proposition 2.3. Let $K \subset T$ be a compact set and $E=\left\{\xi \in \mathbb{R}^{2 \times 2}:\left(\lambda_{1}(\xi), \lambda_{2}(\xi)\right) \in K\right\}$. Let

$$
\begin{aligned}
\Sigma & =\left\{(\theta, \gamma) \in \mathbb{R}_{+}^{2}: \gamma \geq \theta^{2} \text { et } \forall(x, y) \in K y \leq \theta+\frac{\gamma-\theta^{2}}{x+\theta}\right\} \\
\sigma(x) & =\inf _{(\theta, \gamma) \in \Sigma} \theta+\frac{\gamma-\theta^{2}}{x+\theta}, \forall x \geq 0 .
\end{aligned}
$$

Then

$$
\operatorname{Pco} E=\left\{\xi \in \mathbb{R}^{2 \times 2}: \lambda_{2}(\xi) \leq \sigma\left(\lambda_{1}(\xi)\right)\right\} .
$$

It will be useful for our purposes to write in a different way the function $\sigma$ defined by (2.1). We will use the notation $m(\theta)=\max _{(a, b) \in K}\{a b+\theta(b-a)\}$ throughout all this article.

Proposition 2.4. Let $K \subset T$ be a compact set satisfying (1.4) and $\sigma$ be the function defined by (2.1). Then

$$
\sigma(x)=\min _{\theta \in\left[0, \max _{(a, b) \in K} b\right]} \frac{\theta x+m(\theta)}{x+\theta} .
$$

Proof. We divide the proof into two steps: in the first one we study the set $\Sigma$ defined in Proposition 2.3, and in the second one we state the formula for the function $\sigma$.

Step 1. Study of the set $\Sigma$ : by definition

$$
\Sigma=\left\{(\theta, \gamma) \in \mathbb{R}_{+}^{2}: \gamma \geq \theta^{2} \text { et } \forall(a, b) \in K b \leq \theta+\frac{\gamma-\theta^{2}}{a+\theta}\right\} .
$$

As for every $(a, b) \in K$ we have $a>0$, thanks to $(1.4)$

$$
\Sigma=\left\{(\theta, \gamma) \in \mathbb{R}_{+}^{2}: \gamma \geq \sup \left\{\theta^{2}, \max _{(a, b) \in K}\{a b+\theta(b-a)\}\right\}\right\} .
$$

We observe that if $\theta \geq \max _{(a, b) \in K} b$ then for every $(a, b) \in K$ one has

$$
a b+\theta(b-a) \leq \theta a+\theta(b-a)=\theta b \leq \theta^{2}
$$

and so $\max _{(a, b) \in K}\{a b+\theta(b-a)\} \leq \theta^{2}$.

If $\theta<\max _{(a, b) \in K} b$ then, considering $\left(a, \max _{(a, b) \in K} b\right) \in K$ we have

$$
\max _{(a, b) \in K}\{a b+\theta(b-a)\} \geq a\left(\max _{(a, b) \in K} b-\theta\right)+\theta \max _{(a, b) \in K} b>\theta \max _{(a, b) \in K} b>\theta^{2} .
$$

If $\theta=\max _{(a, b) \in K} b$ we have

$$
\max _{(a, b) \in K}\left\{a b+\left[\max _{(a, b) \in K} b\right](b-a)\right\}=\left[\max _{(a, b) \in K} b\right]^{2} .
$$


From this study we can infer that

$$
\Sigma=\Sigma_{1} \cup \Sigma_{2}
$$

where

$$
\begin{aligned}
& \Sigma_{1}=\left\{(\theta, \gamma) \in \mathbb{R}^{2}: \theta \in\left[0, \max _{(a, b) \in K} b\right], \gamma \geq \max _{(a, b) \in K}\{a b+\theta(b-a)\}\right\} \\
& \Sigma_{2}=\left\{(\theta, \gamma) \in \mathbb{R}^{2}: \theta \geq \max _{(a, b) \in K} b, \gamma \geq \theta^{2}\right\} .
\end{aligned}
$$

Step 2. Study of the function $\sigma$ : we define

$$
g_{x}(\theta, \gamma)=\frac{\theta x+\gamma}{x+\theta} \text { for } x \geq 0
$$

and

$$
\begin{aligned}
& \Gamma_{1}=\left\{\left(\theta, \max _{(a, b) \in K}\{a b+\theta(b-a)\}\right), \theta \in\left[0, \max _{(a, b) \in K} b\right]\right\} \\
& \Gamma_{2}=\left\{\left(\theta, \theta^{2}\right), \theta \geq \max _{(a, b) \in K} b\right\} .
\end{aligned}
$$

We observe that $\Gamma_{1} \cup \Gamma_{2} \subset \Sigma_{1} \cup \Sigma_{2}$. We are going to show that

$$
\sigma(x)=\inf _{\Gamma_{1} \cup \Gamma_{2}} g_{x}(\theta, \gamma)
$$

We know from Proposition 2.3 that $\sigma(x)=\inf _{(\theta, \gamma) \in \Sigma} \theta+\frac{\gamma-\theta^{2}}{x+\theta}$. Now, if $x=0$

$$
\sigma(0)=\inf _{(\theta, \gamma) \in \Sigma} \theta+\frac{\gamma-\theta^{2}}{\theta}=\inf _{(\theta, \gamma) \in \Sigma} \frac{\gamma}{\theta}
$$

As $\left(\theta_{0}\right.$ fixed) the function $\frac{\gamma}{\theta_{0}}$ is increasing,

$$
\sigma(0)=\inf _{(\theta, \gamma) \in \Sigma} \frac{\gamma}{\theta}=\inf _{\Gamma_{1} \cup \Gamma_{2}} g_{0}(\theta, \gamma)
$$

If $x>0, \sigma(x)=\inf _{(\theta, \gamma) \in \Sigma} \theta+\frac{\gamma-\theta^{2}}{x+\theta}=\inf _{(\theta, \gamma) \in \Sigma} g_{x}(\theta, \gamma)$. We observe that $\frac{\partial g_{x}}{\partial \gamma}>0:$ this implies that, if $\theta_{0} \geq 0$ is fixed, $g_{x}\left(\theta_{0}, \gamma\right)$ is increasing (in $\left.\gamma\right)$, and so for $x>0$

$$
\sigma(x)=\inf _{\Sigma} g_{x}(\theta, \gamma)=\inf _{\Gamma_{1} \cup \Gamma_{2}} g_{x}(\theta, \gamma)
$$

as wished. We observe that

and so

$$
\left.g_{x}\right|_{\Gamma_{1}}=\frac{\theta x+\max _{(a, b) \in K}\{a b+\theta(b-a)\}}{x+\theta},
$$

$$
\inf _{\Gamma_{1}} g_{x}=\inf _{\theta \in\left[0, \max _{(a, b) \in K} b\right]} \frac{\theta x+\max _{(a, b) \in K}\{a b+\theta(b-a)\}}{x+\theta} .
$$


Moreover

$$
\left.g_{x}\right|_{\Gamma_{2}}=\frac{\theta x+\theta^{2}}{x+\theta}=\theta, \theta \geq \max _{(a, b) \in K} b:
$$

consequently

$$
\inf _{\Gamma_{2}} g_{x}=\max _{(a, b) \in K} b .
$$

This study implies that

$$
\sigma(x)=\inf _{\Gamma_{1} \cup \Gamma_{2}} g_{x}(\theta, \gamma)=\inf \left\{\inf _{\theta \in\left[0, \max _{(a, b) \in K} b\right]} \frac{\theta x+\max _{(a, b) \in K}\{a b+\theta(b-a)\}}{x+\theta}, \max _{(a, b) \in K} b\right\} .
$$

Due to $(2.3)$

$$
\frac{x \max _{(a, b) \in K} b+\max _{(a, b) \in K}\left\{a b+\left[\max _{(a, b) \in K} b\right](b-a)\right\}}{x+\max _{(a, b) \in K} b}=\max _{(a, b) \in K} b
$$

therefore one has

$$
\sigma(x)=\inf _{\theta \in\left[0, \max _{(a, b) \in K} b\right]} \frac{\theta x+\max _{(a, b) \in K}\{a b+\theta(b-a)\}}{x+\theta}=\inf _{\theta \in\left[0, \max _{(a, b) \in K} b\right]} \frac{\theta x+m(\theta)}{x+\theta} .
$$

We are going to show that $\forall x \geq 0$

$$
\inf _{\theta \in\left[0, \max _{(a, b) \in K} b\right]} \frac{\theta x+m(\theta)}{x+\theta}=\min _{\theta \in\left[0, \max _{(a, b) \in K} b\right]} \frac{\theta x+m(\theta)}{x+\theta},
$$

and so the formula of the statement. For $x>0$ this is trivial. For $x=0$ we have to study the function of $\theta$

$$
\frac{m(\theta)}{\theta}=\frac{\max _{(a, b) \in K}\{a b+\theta(b-a)\}}{\theta}:
$$

we observe that,

$$
\frac{\max _{(a, b) \in K}\{a b+\theta(b-a)\}}{\theta} \geq \frac{\max _{(a, b) \in K} a b}{\theta} \rightarrow \infty, \theta \rightarrow 0 .
$$

This implies that there exists $\bar{\varepsilon}>0$ such that

$$
\inf _{\theta \in\left[0, \max _{(a, b) \in K} b\right]} \frac{m(\theta)}{\theta}=\inf _{\theta \in\left[\bar{\varepsilon}, \max _{(a, b) \in K} b\right]} \frac{m(\theta)}{\theta}=\min _{\theta \in\left[\bar{\varepsilon}, \max _{(a, b) \in K} b\right]} \frac{m(\theta)}{\theta},
$$

and so we have the result.

In the next proposition we study the interior of Pco $E$.

Proposition 2.5. Let $K \subset T$ be a compact set satisfying (1.4). Let $E=\left\{\xi \in \mathbb{R}^{2 \times 2}:\left(\lambda_{1}(\xi), \lambda_{2}(\xi)\right) \in K\right\}$. Then, if $\sigma$ is the function defined by (2.2),

$$
\text { int } \mathrm{P} \operatorname{co} E=\left\{\xi \in \mathbb{R}^{2 \times 2}: \lambda_{2}(\xi)<\sigma\left(\lambda_{1}(\xi)\right)\right\}
$$


Proof. The proof is divided into two steps.

Step 1. To show that int $\mathrm{Pco} E \supseteq\left\{\xi \in \mathbb{R}^{2 \times 2}: \lambda_{2}(\xi)<\sigma\left(\lambda_{1}(\xi)\right)\right\}$, as $\lambda_{i}(\xi)$ are continuous functions, $i=1,2$, it is sufficient to show that the function $\sigma$ is continuous for $x \geq 0$. This is easy for $x>0$. To study the point $x=0$, we are going to show that if $x_{n} \rightarrow 0^{+}$, then $\sigma\left(x_{n}\right) \rightarrow \sigma(0)$. We can say from (2.4) that there exists $\theta_{0} \in\left(0, \max _{(a, b) \in K} b\right]$ such that $\sigma(0)=\frac{m\left(\theta_{0}\right)}{\theta_{0}}$. Then, by definition of $\sigma\left(x_{n}\right)$

$$
\sigma\left(x_{n}\right)-\sigma(0) \leq \frac{\theta_{0} x_{n}+m\left(\theta_{0}\right)}{x_{n}+\theta_{0}}-\frac{m\left(\theta_{0}\right)}{\theta_{0}} \rightarrow 0, n \rightarrow \infty
$$

We are going to show that $\sigma\left(x_{n}\right)-\sigma(0) \geq h\left(x_{n}\right)$, with $h\left(x_{n}\right) \rightarrow 0$, if $n \rightarrow \infty$ : this will imply that $\sigma\left(x_{n}\right) \rightarrow \sigma(0)$, and so the continuity of $\sigma$ in 0 .

For every $x_{n}$ there exists $\theta_{n} \in\left[0, \max _{(a, b) \in K} b\right]$ such that

$$
\sigma\left(x_{n}\right)=\frac{\theta_{n} x_{n}+m\left(\theta_{n}\right)}{x_{n}+\theta_{n}}
$$

Then, by definition of $\sigma(0)$

$$
\begin{aligned}
\sigma\left(x_{n}\right)-\sigma(0) & =\frac{\theta_{n} x_{n}+m\left(\theta_{n}\right)}{x_{n}+\theta_{n}}-\min _{\theta \in\left[0, \max _{(a, b) \in K} b\right]} \frac{m(\theta)}{\theta} \\
& \geq \frac{\theta_{n} x_{n}+m\left(\theta_{n}\right)}{x_{n}+\theta_{n}}-\frac{m\left(\theta_{n}\right)}{\theta_{n}}=\frac{x_{n}}{\theta_{n}\left(x_{n}+\theta_{n}\right)}\left[\theta_{n}^{2}-m\left(\theta_{n}\right)\right] .
\end{aligned}
$$

We will show that $h\left(x_{n}\right)=\frac{x_{n}}{\theta_{n}\left(x_{n}+\theta_{n}\right)}\left[\theta_{n}^{2}-m\left(\theta_{n}\right)\right] \rightarrow 0, n \rightarrow \infty$. As $\theta_{n}^{2}-m\left(\theta_{n}\right)$ is bounded, it is now sufficient to prove that

$$
\frac{x_{n}}{\theta_{n}\left(x_{n}+\theta_{n}\right)} \rightarrow 0, n \rightarrow \infty
$$

We observe that $\liminf _{n \rightarrow \infty} \theta_{n}>0$. In fact, if $\liminf _{n \rightarrow \infty} \theta_{n}=0$, then there exists a sub-sequence $n_{k}$ such that $\lim _{k \rightarrow \infty} \theta_{n_{k}}=$ 0 ; consequently

The matrix

$$
\sigma\left(x_{n_{k}}\right)=\frac{\theta_{n_{k}} x_{n_{k}}+m\left(\theta_{n_{k}}\right)}{x_{n_{k}}+\theta_{n_{k}}} \geq \frac{\max _{(a, b) \in K} a b}{x_{n_{k}}+\theta_{n_{k}}} \rightarrow \infty, k \rightarrow \infty .
$$

$$
\xi_{n_{k}}=\left(\begin{array}{cc}
x_{n_{k}} & 0 \\
0 & \sigma\left(x_{n_{k}}\right)
\end{array}\right)
$$

belongs to Pco $E$ and $\lambda_{2}\left(\xi_{n_{k}}\right) \rightarrow \infty$ : recalling that $\lambda_{2}$ is a norm over $\mathbb{R}^{2 \times 2}$ (see [6]), we got a contradiction because $\mathrm{P} \operatorname{co} E$ is bounded, as $E$ is bounded.

Then $\liminf _{n \rightarrow \infty} \theta_{n}=a>0$ : this implies that, for $n$ sufficiently large $\theta_{n} \geq \frac{a}{2}$ and so

$$
\frac{x_{n}}{\theta_{n}\left(x_{n}+\theta_{n}\right)} \leq \frac{x_{n}}{\frac{a}{2}\left(x_{n}+\frac{a}{2}\right)} \rightarrow 0, n \rightarrow \infty,
$$

that is the result.

Step 2. We now show that int Pco $E \subseteq\left\{\xi \in \mathbb{R}^{2 \times 2}: \lambda_{2}(\xi)<\sigma\left(\lambda_{1}(\xi)\right)\right\}$. Suppose that there exists a matrix $\eta \in \operatorname{int} \operatorname{Pco} E$ such that $\lambda_{2}(\eta)=\sigma\left(\lambda_{1}(\eta)\right)$; therefore the ball $B_{\varepsilon}(\eta) \subseteq \operatorname{Pco} E$, for some $\varepsilon>0$. Let $A, B \in \mathcal{O}(2)$ be such that

$$
A \eta B=\left(\begin{array}{cc}
\lambda_{1}(\eta) & 0 \\
0 & \lambda_{2}(\eta)
\end{array}\right)
$$


we define

$$
D=A^{-1}\left(\begin{array}{cc}
0 & 0 \\
0 & d
\end{array}\right) B^{-1}
$$

with $0<d<\varepsilon$. Then we have

$$
\begin{aligned}
& \lambda_{1}(\eta+D)=\lambda_{1}(A \eta B+A D B)=\lambda_{1}(\eta) \\
& \lambda_{2}(\eta+D)=\lambda_{2}(A \eta B+A D B)=\lambda_{2}(\eta)+d
\end{aligned}
$$

The matrix $\eta+D \in B_{\varepsilon}(\eta) \subseteq \mathrm{P} \operatorname{co} E$, as $d<\varepsilon$ : this implies that $\lambda_{2}(\eta+D) \leq \sigma\left(\lambda_{1}(\eta+D)\right)$. From other hand,

$$
\lambda_{2}(\eta+D)=\lambda_{2}(\eta)+d=\sigma\left(\lambda_{1}(\eta)\right)+d=\sigma\left(\lambda_{1}(\eta+D)\right)+d>\sigma\left(\lambda_{1}(\eta+D)\right),
$$

and this is a contradiction: therefore $\lambda_{2}(\eta)<\sigma\left(\lambda_{1}(\eta)\right)$.

Remark 2.6. The previous results imply that if $\xi \in \partial \mathrm{P} \operatorname{co} E$, then there exists $\bar{\theta} \in[0, \underset{(a, b) \in K}{\max } b]$ such that

$$
\lambda_{1}(\xi) \lambda_{2}(\xi)+\bar{\theta}\left(\lambda_{2}(\xi)-\lambda_{1}(\xi)\right)=m(\bar{\theta}) .
$$

\section{The RANK One CONVEX Hull}

In this section we are going to prove the representation theorem of the rank one convex hull of $E$. It will be useful to show our existence result. We recall that we use the notation $m(\theta)=\max _{(a, b) \in K}\{a b+\theta(b-a)\}$.

Theorem 3.1. Let $K \subset T$ be a compact set satisfying (1.4). Let

$$
E=\left\{\xi \in \mathbb{R}^{2 \times 2}:\left(\lambda_{1}(\xi), \lambda_{2}(\xi)\right) \in K\right\} .
$$

Then

$$
\begin{aligned}
\operatorname{Rco} E & =\left\{\xi \in \mathbb{R}^{2 \times 2}: \lambda_{1}(\xi) \lambda_{2}(\xi)+\theta\left(\lambda_{2}(\xi)-\lambda_{1}(\xi)\right) \leq m(\theta), \forall \theta \in\left[0, \max _{(a, b) \in K} b\right]\right\}, \\
\operatorname{int} \operatorname{Rco} E & =\left\{\xi \in \mathbb{R}^{2 \times 2}: \lambda_{1}(\xi) \lambda_{2}(\xi)+\theta\left(\lambda_{2}(\xi)-\lambda_{1}(\xi)\right)<m(\theta), \forall \theta \in\left[0, \max _{(a, b) \in K} b\right]\right\} .
\end{aligned}
$$

We will first prove the following lemma:

Lemma 3.2. Let $K=\left\{\left(a_{1}, b_{1}\right),\left(a_{2}, b_{2}\right)\right\}, 0<a_{1}<a_{2}, a_{1} b_{1} \leq a_{2} b_{2}, b_{2} \leq b_{1}, a_{1} \leq b_{1}, a_{2} \leq b_{2}$ and $E=\{\xi \in$ $\left.\mathbb{R}^{2 \times 2}:\left(\lambda_{1}(\xi), \lambda_{2}(\xi)\right) \in K\right\}$. Then

$$
\begin{aligned}
\operatorname{Rco} E=\left\{\xi \in \mathbb{R}^{2 \times 2}:\right. & \lambda_{2}(\xi) \leq b_{1}, \\
& \lambda_{1}(\xi) \lambda_{2}(\xi) \leq a_{2} b_{2} \\
& \left.\lambda_{1}(\xi) \lambda_{2}(\xi)+\bar{\theta}\left(\lambda_{2}(\xi)-\lambda_{1}(\xi)\right) \leq a_{1} b_{1}+\bar{\theta}\left(b_{1}-a_{1}\right)\right\}
\end{aligned}
$$

where $\bar{\theta}=\frac{a_{2} b_{2}-a_{1} b_{1}}{b_{1}-a_{1}-b_{2}+a_{2}}$.

Remark 3.3. We remember that Dacorogna and Marcellini [3] proved that if $K$ is composed by one point $(a, b)$ we have

$$
\operatorname{Rco} E=\left\{\xi \in \mathbb{R}^{2 \times 2}: \lambda_{2}(\xi) \leq b, \lambda_{1}(\xi) \lambda_{2}(\xi) \leq a b\right\} .
$$


Proof. In [1] it is showed that the function $\sigma(2.2)$ defined for $E$ is

$$
\sigma(x)=\inf \left\{b_{1}, \frac{a_{2} b_{2}}{x}, \frac{\bar{\theta} x+a_{1} b_{1}+\bar{\theta}\left(b_{1}-a_{1}\right)}{\bar{\theta}+x}\right\} .
$$

Thanks to Proposition 2.3, this implies that Pco $E$ is the set of matrices $\xi$ such that

$$
\begin{aligned}
& \lambda_{2}(\xi) \leq b_{1}, \\
& \lambda_{1}(\xi) \lambda_{2}(\xi) \leq a_{2} b_{2} \\
& \lambda_{1}(\xi) \lambda_{2}(\xi)+\bar{\theta}\left(\lambda_{2}(\xi)-\lambda_{1}(\xi)\right) \leq a_{1} b_{1}+\bar{\theta}\left(b_{1}-a_{1}\right) .
\end{aligned}
$$

Therefore to prove the formula of $\mathrm{R} c o E$ it is sufficient to prove that $\mathrm{P} \operatorname{co} E=\mathrm{R} \operatorname{co} E$. For this we will show that

$$
\partial \mathrm{P} c o E \subseteq \mathrm{R} c o E:
$$

this will imply the not trivial inclusion $\mathrm{P} \operatorname{co} E \subseteq \mathrm{R} \operatorname{co} E$ and so the result. In fact, let $\xi \in \operatorname{intP} \operatorname{co} E$; as $\mathrm{P} \operatorname{co} E$ is compact, then for any rank one matrix $\lambda \in \mathbb{R}^{2 \times 2}$, there exist $t_{1}=t_{1}(\lambda)<0<t_{2}=t_{2}(\lambda)$ such that $\xi+t_{i} \lambda \in \partial \mathrm{P} \operatorname{co} E \subseteq \operatorname{Rco} E, i=1,2$. Defining $\xi_{i}=\xi+t_{i} \lambda, i=1,2$, we have

$$
\xi=\frac{t_{2}}{t_{2}-t_{1}} \xi_{1}-\frac{t_{1}}{t_{2}-t_{1}} \xi_{2} \in \mathrm{Rco} E
$$

because $\operatorname{rk}\left(\xi_{1}-\xi_{2}\right)=1$.

Now, let $\xi \in \partial \mathrm{P} c o E$ : necessarily $\lambda_{2}(\xi)=\sigma\left(\lambda_{1}(\xi)\right)$ and so $\xi$ satisfies either (3.1) or (3.2) or (3.3) with equality. We are going to treat these cases separately (steps $1-3$ respectively) to show that $\xi \in \mathrm{Rco} E$. We can assume without loss of generality that $\xi=\operatorname{diag}\left(\lambda_{1}(\xi), \lambda_{2}(\xi)\right)$, as Rco $E$ is isotropic: in fact, using the same notations as in the second point of Remark 2.2, we have by induction on $i$ that $\mathrm{R}_{i} \operatorname{co} E$ is isotropic and so $\mathrm{Rco} E$ is isotropic.

Step 1. If $\xi$ satisfies (3.1) with equality, (3.3) implies that $\lambda_{1}(\xi) \leq a_{1}$; then $\xi \in \operatorname{Rco} E$, as

$$
\xi=\left(\begin{array}{cc}
\lambda_{1}(\xi) & 0 \\
0 & b_{1}
\end{array}\right)=t\left(\begin{array}{cc}
a_{1} & 0 \\
0 & b_{1}
\end{array}\right)+(1-t)\left(\begin{array}{cc}
-a_{1} & 0 \\
0 & b_{1}
\end{array}\right), t \in(0,1)
$$

In the next steps we can assume that $\xi$ satisfies (3.1) with strict inequality.

Step 2. We suppose that $\xi$ satisfies (3.2) with equality. Moreover we can assume that $\xi$ satisfies (3.3) with strict inequality, otherwise these two equalities imply

$$
\xi=\left(\begin{array}{cc}
a_{2} & 0 \\
0 & b_{2}
\end{array}\right) \in E
$$

If we define

$$
V=\left\{\xi \in \mathbb{R}^{2 \times 2}: \lambda_{1}(\xi) \lambda_{2}(\xi)=a_{2} b_{2}\right\}, Y=V \cap \partial \mathrm{P} \operatorname{co} E
$$

we have that $\xi \in \operatorname{rel} \operatorname{int} Y^{1}$. Let $Z$ be the rank one matrix defined by

$$
Z=\left(\begin{array}{rr}
1 & -\frac{\lambda_{2}(\xi)}{\lambda_{1}(\xi)} \\
1 & -\frac{\lambda_{2}(\xi)}{\lambda_{1}(\xi)}
\end{array}\right):
$$

\footnotetext{
${ }^{1}$ Relative interior of $Y$.
} 
then $\lambda_{1}(\xi+t Z) \lambda_{2}(\xi+t Z)=\lambda_{1}(\xi) \lambda_{2}(\xi)=a_{2} b_{2} \forall t \in \mathbb{R}$. This implies, as $Y$ is compact, that there exist $t_{1}<0<$ $t_{2}: \xi+t_{i} Z \in \partial Y, i=1,2$. Consequently $\xi+t_{i} Z$ satisfies either (3.1) and (3.2) as equalities or (3.3) and (3.2) as equalities: from the previous studies we obtain that $\xi+t_{i} Z \in \operatorname{Rco} E, i=1,2$ and so $\xi \in \operatorname{Rco} E$.

In the next step we can assume that (3.1) and (3.2) are satisfied as strict inequalities.

Step 3. We assume that $\xi$ satisfies (3.3) with equality. Using the explicit expressions of $\lambda_{1}, \lambda_{2}($ see $(1.3))$, it is easy to prove that if $\left(\lambda_{1}(\xi), \lambda_{2}(\xi)\right)=(x, y)$ the matrix defined by

$$
A=\left(\begin{array}{cc}
1 & \sqrt{\frac{y-\bar{\theta}}{x+\bar{\theta}}} \\
-\sqrt{\frac{y-\bar{\theta}}{x+\bar{\theta}}} & -\frac{y-\bar{\theta}}{x+\bar{\theta}}
\end{array}\right)
$$

has the following properties: it is well defined (as $y \geq \bar{\theta}$ because $\left.\bar{\theta}^{2} \leq \max _{(a, b) \in K} a b+\bar{\theta}(b-a)=x y+\bar{\theta}(y-x)\right)$ and

$$
\begin{aligned}
& \lambda_{1}(\xi+t A) \lambda_{2}(\xi+t A)+\bar{\theta}\left[\lambda_{2}(\xi+t A)-\lambda_{1}(\xi+t A)\right]=x y+\bar{\theta}(y-x) \\
& \forall t \in\left[t_{-}, t_{+}\right], t_{-}=-\frac{x y(x+\bar{\theta})}{\bar{\theta}(x+y)}, t_{+}=\frac{(y-x)(x+\bar{\theta})}{x+y} .
\end{aligned}
$$

In fact

$$
\begin{aligned}
\lambda_{1}(\xi+t A) \lambda_{2}(\xi+t A) & =|\operatorname{det}(\xi+t A)|=\left|x y-x t \frac{y-\bar{\theta}}{x+\bar{\theta}}+t y\right| \\
\left(\lambda_{2}(\xi+t A)-\lambda_{1}(\xi+t A)\right)^{2} & =\|\xi+t A\|^{2}-2|\operatorname{det}(\xi+t A)| \\
& =(x+t)^{2}+\left(y-t \frac{y-\bar{\theta}}{x+\bar{\theta}}\right)^{2}+2 t^{2} \frac{y-\bar{\theta}}{x+\bar{\theta}}-2\left|x y-x t \frac{y-\bar{\theta}}{x+\bar{\theta}}+t y\right| .
\end{aligned}
$$

If we assume that $x y-x t \frac{y-\bar{\theta}}{x+\theta}+t y \geq 0$ (that is $t \geq t_{-}$) (3.4) is equivalent to show that

$$
\bar{\theta}(y-x)+x t \frac{y-\bar{\theta}}{x+\bar{\theta}}-t y=\bar{\theta} \sqrt{\|\xi+t A\|^{2}-2[\operatorname{det}(\xi+t A)]} .
$$

If we assume that $\bar{\theta}(y-x)+x t \frac{y-\bar{\theta}}{x+\theta}-y t \geq 0$ (that is $\left.t \leq t_{+}\right)$we get

$$
\begin{aligned}
t^{2} x^{2}\left(\frac{y-\bar{\theta}}{x+\bar{\theta}}\right)^{2}+t^{2} y^{2}+2 \bar{\theta}(y-x) x t \frac{y-\bar{\theta}}{x+\bar{\theta}}-2 \bar{\theta}(y-x) y t-2 t^{2} x y \frac{y-\bar{\theta}}{x+\bar{\theta}}= \\
\bar{\theta}^{2}\left[t^{2}+2 x t+t^{2}\left(\frac{y-\bar{\theta}}{x+\bar{\theta}}\right)^{2}-2 y t \frac{y-\bar{\theta}}{x+\bar{\theta}}+2 t^{2} \frac{y-\bar{\theta}}{x+\bar{\theta}}+2 x t \frac{y-\bar{\theta}}{x+\bar{\theta}}-2 y t\right] .
\end{aligned}
$$

One can easily check that this equality is verified for every $t$ and so (3.4) is verified.

We prove now that there exists $t_{1} \in\left[t_{-}, 0\right]$ such that $\lambda_{2}\left(\xi+t_{1} A\right)=b_{1}$ : this implies that $\xi+t_{1} A$ satisfies $(3.1)$ and (3.3) as equalities: as we saw in the first step, $\xi+t_{1} A \in \mathrm{Rco} E$. Moreover we prove also that there exists $t_{2} \in\left[0, t_{+}\right]$such that $\lambda_{1}\left(\xi+t_{2} A\right) \lambda_{2}\left(\xi+t_{2} A\right)=a_{2} b_{2}$ : this implies that $\xi+t_{2} A$ satisfies (3.2) and (3.3) as equalities: as we saw in the second step, $\xi+t_{2} A \in \mathrm{Rco} E$. Consequently $\xi \in \mathrm{Rco} E$, as it can be written as rank one combination of $\xi+t_{1} A$ and $\xi+t_{2} A$. 
Existence of $t_{1}$. We consider $F(t)=\lambda_{2}(\xi+t A)-b_{1}$. The existence of $t_{1}$ follows from the fact that this function is continuous and $F(0)<0<F\left(t_{-}\right)$: in fact

$$
\begin{aligned}
F\left(t_{-}\right) & =\left\|\xi+t_{-} A\right\|-b_{1}>0 \Longleftrightarrow \\
b_{1}<\left\|\xi+t_{-} A\right\| & =\sqrt{\left(\frac{x^{2}(\bar{\theta}-y)}{\bar{\theta}(x+y)}\right)^{2}+\left(\frac{y^{2}(\bar{\theta}+x)}{\bar{\theta}(x+y)}\right)^{2}+2 \frac{x^{2} y^{2}(x+\bar{\theta})(y-\bar{\theta})}{\bar{\theta}^{2}(x+y)^{2}}} \\
& =\frac{x^{2}(y-\bar{\theta})+y^{2}(\bar{\theta}+x)}{\bar{\theta}(x+y)}=\frac{x y+\bar{\theta}(y-x)}{\bar{\theta}} .
\end{aligned}
$$

The last inequality is equivalent to

$$
\bar{\theta} b_{1} \leq x y+\bar{\theta}(y-x)=a_{1} b_{1}+\bar{\theta}\left(b_{1}-a_{1}\right) \Longleftrightarrow b_{1} \geq \bar{\theta}
$$

which is true.

Existence of $t_{2}$. We consider $G(t)=\lambda_{1}(\xi+t A) \lambda_{2}(\xi+t A)-a_{2} b_{2}$. The existence of $t_{2}$ follows from the fact that $G$ is continuous and $G(0)<0<G\left(t_{+}\right)$: in fact $G\left(t_{+}\right)>0$ if and only if

$$
\left|x y-x \frac{(y-\bar{\theta})(y-x)}{x+y}+y \frac{(x+\bar{\theta})(y-x)}{x+y}\right| \geq a_{2} b_{2} .
$$

Using that $x y+\bar{\theta}(y-x)=a_{2} b_{2}+\bar{\theta}\left(b_{2}-a_{2}\right)$ we get

$$
x y(x+y)+\bar{\theta}(y-x)(y+x) \geq a_{2} b_{2}(x+y) \Longleftrightarrow a_{2} b_{2}+\bar{\theta}\left(b_{2}-a_{2}\right) \geq a_{2} b_{2}
$$

which is true.

To prove Theorem 3.1 it will be useful to recall the following properties about convex functions and their sub-differential (we will follow the definition of [9]).

Definition 3.4. Let $f: \mathbb{R} \rightarrow \mathbb{R}$ be a convex function and $\bar{\theta} \in \mathbb{R}$. The sub-differential of $f$ in $\bar{\theta}$ is the set

$$
\partial f(\bar{\theta})=\left\{\theta^{*} \in \mathbb{R}: f(\theta) \geq f(\bar{\theta})+\theta^{*}(\theta-\bar{\theta}) \quad \forall \theta \in \mathbb{R}\right\} .
$$

Proposition 3.5. Let $f: \mathbb{R} \rightarrow \mathbb{R}$ be a convex function. Then

i) $\partial f(\theta)$ is non empty, compact and convex for every $\theta \in \mathbb{R}$.

ii) If $\theta$ is a point of differentiability of $f$ then $\partial f(\theta)=\left\{f^{\prime}(\theta)\right\}$.

iii) The set of points of differentiability of $f$ is dense in $\mathbb{R}$ and

$$
\partial f(x)=\operatorname{co} \overline{S(x)}, S(x)=\left\{\lim _{n \rightarrow \infty} f^{\prime}\left(x_{n}\right) \text { fdifferentiable in } x_{n}, x_{n} \rightarrow x\right\} .
$$

Remark 3.6. The proof of $i$ ) can be found at page 218 of [9]; ii) is Theorem 26.1 of [9]; the proof of iii) is a direct combination of Theorems 25.6 and 17.2 of [9].

We pass now to the proof of Theorem 3.1.

Proof. Thanks to Propositions 2.3 and 2.4 it is sufficient to prove that Pco $E=$ Rco E. For this, as in Lemma 3.2, we will show the inclusion

$$
\partial \mathrm{P} \operatorname{co} E \subseteq \mathrm{R} \operatorname{co} E .
$$

Let $\xi \in \partial \mathrm{Pco} E$. We have seen in Remark 2.6 that there exists $\bar{\theta} \in\left[0, \max _{(a, b) \in K} b\right]$ such that

$$
\lambda_{1}(\xi) \lambda_{2}(\xi)+\bar{\theta}\left(\lambda_{2}(\xi)-\lambda_{1}(\xi)\right)=m(\bar{\theta}),
$$


and for every $\theta \in\left[0, \max _{(a, b) \in K} b\right]$

$$
\lambda_{1}(\xi) \lambda_{2}(\xi)+\theta\left(\lambda_{2}(\xi)-\lambda_{1}(\xi)\right) \leq m(\theta)
$$

We define

$$
F(\theta)= \begin{cases}\max _{(a, b) \in K} a b & \theta \leq 0 \\ m(\theta) & \theta \in\left[0, \max _{(a, b) \in K} b\right] \\ \theta^{2} & \theta \geq \max _{(a, b) \in K} b .\end{cases}
$$

We observe that $\xi$ satisfies

$$
\lambda_{1}(\xi) \lambda_{2}(\xi)+\theta\left(\lambda_{2}(\xi)-\lambda_{1}(\xi)\right) \leq F(\theta), \quad \forall \theta \in \mathbb{R},
$$

and there exists $\bar{\theta} \in\left[0, \max _{(a, b) \in K} b\right]$ such that

$$
\lambda_{1}(\xi) \lambda_{2}(\xi)+\bar{\theta}\left(\lambda_{2}(\xi)-\lambda_{1}(\xi)\right)=F(\bar{\theta}) .
$$

The following remarks will be useful:

- $\lambda_{2}(\xi)-\lambda_{1}(\xi) \in \partial F(\bar{\theta})$.

- One can easily check that $F$ is convex: the previous proposition implies that $\partial F(\bar{\theta})=[\alpha(\bar{\theta}), \beta(\bar{\theta})]$ for some $\alpha(\bar{\theta}), \beta(\bar{\theta}) \in \mathbb{R}$.

- If $\theta_{0}$ is a point of differentiability for the function $F$ then $\partial F\left(\theta_{0}\right)=\left\{F^{\prime}\left(\theta_{0}\right)\right\}=b_{0}-a_{0}$ for some $\left(a_{0}, b_{0}\right) \in K$, such that $F\left(\theta_{0}\right)=\max _{(a, b) \in K}\left\{a b+\theta_{0}(b-a)\right\}=a_{0} b_{0}+\theta_{0}\left(b_{0}-a_{0}\right)$.

We are going to show that $\xi \in$ Rco $E$ : for this we will find a set $a \subset K$ composed by one or two points such that letting $A=\left\{\xi \in \mathbb{R}^{2 \times 2}:\left(\lambda_{1}(\xi), \lambda_{2}(\xi)\right) \in a\right\}$ we have $\xi \in \operatorname{Rco} A \subseteq \operatorname{Rco} E$. We will distinguish the cases $\bar{\theta}=0, \bar{\theta} \in\left(0, \max _{(a, b) \in K} b\right), \bar{\theta}=\max _{(a, b) \in K} b$ respectively in the steps 1-3.

Step 1. We analyse the case $\bar{\theta}=0$, for which $\max _{(a, b) \in K} a b=\lambda_{1}(\xi) \lambda_{2}(\xi)$. We study the set $S(0)$ defined in Proposition 3.5 (the points $\theta_{n}$ will be points of differentiability of $F$ throughout this proof):

$$
S(0)=\left\{\lim _{n \rightarrow \infty} F^{\prime}\left(\theta_{n}\right), \theta_{n} \rightarrow 0\right\}=\left\{\lim _{n \rightarrow \infty} F^{\prime}\left(\theta_{n}\right), \theta_{n} \rightarrow 0^{+}\right\} \cup\{0\}
$$

as for every $\theta<0 F$ is constant. Let

$$
p_{M}=\sup \left\{\lim _{n \rightarrow \infty} F^{\prime}\left(\theta_{n}\right), \theta_{n} \rightarrow 0^{+}\right\} \geq 0 .
$$

Let $\theta_{n} \rightarrow 0^{+}$be points of differentiability for $F$ : then $F^{\prime}\left(\theta_{n}\right)=b_{n}-a_{n}$, for some $\left(a_{n}, b_{n}\right) \in K$; therefore every point of $S(0)$ is 0 or $b-a$ for some $(a, b) \in K$, because of the compactness of $K$. The fact that $K$ is compact implies also that $p_{M}=\bar{b}-\bar{a}$, for some $(\bar{a}, \bar{b}) \in K$ and so

$$
\partial F(0)=\cos \overline{S(0)}=[0, \bar{b}-\bar{a}] \ni \lambda_{2}(\xi)-\lambda_{1}(\xi) .
$$

It is easy to see that there exists $(\tilde{a}, \tilde{b}) \in K$ such that $\bar{b}-\bar{a}=\tilde{b}-\tilde{a}$ and $\max _{(a, b) \in K} a b=\tilde{a} \tilde{b}$. In fact by definition of $\bar{b}-\bar{a}, \forall \varepsilon>0$ there exists $\theta_{n}^{\varepsilon}$ which goes to 0 for $n \rightarrow \infty$ and a sequence $\left(a_{n}^{\varepsilon}, b_{n}^{\varepsilon}\right) \in K$ such that

$$
\bar{b}-\bar{a}-\varepsilon \leq \lim _{n \rightarrow \infty} F^{\prime}\left(\theta_{n}^{\varepsilon}\right)=\lim _{n \rightarrow \infty} b_{n}^{\varepsilon}-a_{n}^{\varepsilon} \leq \bar{b}-\bar{a} .
$$


We observe that, as $\theta_{n}^{\varepsilon}$ is a point of differentiability of $F$

$$
\max _{(a, b) \in K} a b+\theta_{n}^{\varepsilon}(b-a)=a_{n}^{\varepsilon} b_{n}^{\varepsilon}+\theta_{n}^{\varepsilon}\left(b_{n}^{\varepsilon}-a_{n}^{\varepsilon}\right)
$$

Now, if we consider the points $\left(a_{n}^{\varepsilon}, b_{n}^{\varepsilon}\right) \in K$, as $K$ is compact, we can say, up to a sub-sequence that $\left(a_{n}^{\varepsilon}, b_{n}^{\varepsilon}\right) \rightarrow$ $\left(a^{\varepsilon}, b^{\varepsilon}\right) \in K$. For the same reason, if $\varepsilon \rightarrow 0\left(a^{\varepsilon}, b^{\varepsilon}\right) \rightarrow(\tilde{a}, \tilde{b}) \in K$. Passing to the limit for $n \rightarrow \infty$ in the last relation, we obtain from the continuity in $\theta$ of the function $\max _{(a, b) \in K} a b+\theta(b-a)$

$$
\lim _{n \rightarrow \infty} \max _{(a, b) \in K} a b+\theta_{n}^{\varepsilon}(b-a)=\max _{(a, b) \in K} a b=\lim _{n \rightarrow \infty} a_{n}^{\varepsilon} b_{n}^{\varepsilon}+\theta_{n}^{\varepsilon}\left(b_{n}^{\varepsilon}-a_{n}^{\varepsilon}\right)=a^{\varepsilon} b^{\varepsilon}
$$

and so

$$
\max _{(a, b) \in K} a b=\lambda_{1}(\xi) \lambda_{2}(\xi)=\lim _{\varepsilon \rightarrow 0} a^{\varepsilon} b^{\varepsilon}=\tilde{a} \tilde{b}
$$

From the relation (3.5) we get,

$$
\bar{b}-\bar{a} \leq \lim _{\varepsilon \rightarrow 0} \lim _{n \rightarrow \infty} b_{n}^{\varepsilon}-a_{n}^{\varepsilon}=\tilde{b}-\tilde{a} \leq \bar{b}-\bar{a} \Longleftrightarrow \bar{b}-\bar{a}=\tilde{b}-\tilde{a} .
$$

Then we have that $\lambda_{1}(\xi) \lambda_{2}(\xi)=\tilde{a} \tilde{b}$ and $\lambda_{2}(\xi)-\lambda_{1}(\xi) \leq \tilde{b}-\tilde{a}$, that is $\lambda_{2}(\xi) \leq \tilde{b}$. This is equivalent, thanks to Remark 3.3, to say that $\xi \in \operatorname{Rco} A \subseteq \operatorname{Rco} E$, where

$$
A=\left\{\xi \in \mathbb{R}^{2 \times 2}:\left(\lambda_{1}(\xi), \lambda_{2}(\xi)\right)=(\tilde{a}, \tilde{b})\right\}
$$

Step 2. We study the case $\bar{\theta} \in\left(0, \max _{(a, b) \in K} b\right)$. As in step 1 , if

$$
\begin{gathered}
p_{m}=\inf \left\{\lim _{n \rightarrow \infty} F^{\prime}\left(\theta_{n}\right), \theta_{n} \rightarrow \bar{\theta}\right\} \\
p_{M}=\sup \left\{\lim _{n \rightarrow \infty} F^{\prime}\left(\theta_{n}\right), \theta_{n} \rightarrow \bar{\theta}\right\},
\end{gathered}
$$

we have for some $\left(a_{i}, b_{i}\right) \in K, i=1,2$

$$
\partial F(\bar{\theta})=\left[b_{2}-a_{2}, b_{1}-a_{1}\right]
$$

Following the same kind of study as in step 1 , one can show that there exist $\left(\tilde{a}_{i}, \tilde{b}_{i}\right) \in K, i=1,2$ such that

$$
\lambda_{1}(\xi) \lambda_{2}(\xi)+\bar{\theta}\left(\lambda_{2}(\xi)-\lambda_{1}(\xi)\right)=F(\bar{\theta})=\max _{(a, b) \in K}\{a b+\bar{\theta}(b-a)\}=\tilde{a}_{i} \tilde{b_{i}}+\bar{\theta}\left(\tilde{b_{i}}-\tilde{a_{i}}\right), \quad \tilde{b_{i}}-\tilde{a}_{i}=b_{i}-a_{i}
$$

We now show that $\xi \in \operatorname{Rco} A$, where

$$
A=\left\{\xi \in \mathbb{R}^{2 \times 2}:\left(\lambda_{1}(\xi), \lambda_{2}(\xi)\right)=\left(\tilde{a_{i}}, \tilde{b}_{i}\right), i=1,2\right\}:
$$

for this, thanks to Lemma 3.2, it is sufficient to show that

$$
\begin{aligned}
& \tilde{b_{2}} \leq \lambda_{2}(\xi) \leq \tilde{b_{1}}, \\
& \tilde{a_{1}} \tilde{b_{1}} \leq \lambda_{1}(\xi) \lambda_{2}(\xi) \leq \tilde{a_{2}} \tilde{b_{2}}, \\
& \tilde{a_{1}}<\tilde{a_{2}} .
\end{aligned}
$$


For every $\theta \in\left[0, \max _{(a, b) \in K} b\right]$ we have that $\theta^{2} \leq \max _{(a, b) \in K} a b+\theta(b-a)$, as seen in Proposition 2.4: writing this inequality for $\theta=\bar{\theta}$ we get $\lambda_{2}(\xi)>\bar{\theta}$. As

$$
\tilde{a_{2}} \tilde{b_{2}}+\theta\left(\tilde{b_{2}}-\tilde{a_{2}}\right)<\lambda_{1}(\xi) \lambda_{2}(\xi)+\theta\left(\lambda_{2}(\xi)-\lambda_{1}(\xi)\right)<\tilde{a_{1}} \tilde{b_{1}}+\theta\left(\tilde{b_{1}}-\tilde{a_{1}}\right)
$$

for every $\theta \geq \bar{\theta}$ and in particular for $\theta=\lambda_{2}(\xi)$ we have that $\tilde{b_{2}} \leq \lambda_{2}(\xi) \leq \tilde{b_{1}}$, that is the first condition is verified. As $\tilde{b_{2}}-\tilde{a_{2}} \leq \lambda_{2}(\xi)-\lambda_{1}(\xi) \leq \tilde{b_{1}}-\tilde{a_{1}}$, then $\tilde{a_{1}} \tilde{b_{1}} \leq \lambda_{1}(\xi) \lambda_{2}(\xi) \leq \tilde{a_{2}} \tilde{b_{2}}$ and $\tilde{a_{1}}<\tilde{a_{2}}$.

Step 3. We study the case $\bar{\theta}=\max _{(a, b) \in K} b$ : we have, as we saw in Proposition $2.4 \lambda_{2}(\xi)=\max _{(a, b) \in K} b$. We define

$$
p_{m}=\inf \left\{\lim _{n \rightarrow \infty} F^{\prime}\left(\theta_{n}\right), \theta_{n} \rightarrow \max _{(a, b) \in K} b\right\}
$$

and we have, as in the previous steps

$$
\partial F(\bar{\theta})=[\bar{b}-\bar{a}, \beta(\bar{\theta})],(\bar{a}, \bar{b}) \in K .
$$

It is easy to show that there exists $(\tilde{a}, \tilde{b}) \in K$ such that $\bar{b}-\bar{a}=\tilde{b}-\tilde{a}$ and $\max _{(a, b) \in K} a b+\bar{\theta}(b-a)=\left[\max _{(a, b) \in K} b\right]^{2}=$ $\tilde{a} \tilde{b}+\bar{\theta}(\tilde{b}-\tilde{a})$. Therefore $\tilde{b}=\max _{(a, b) \in K} b$. Defining

$$
A=\left\{\xi \in \mathbb{R}^{2 \times 2}:\left(\lambda_{1}(\xi), \lambda_{2}(\xi)\right)=(\tilde{a}, \tilde{b})\right\}
$$

we have that $\xi \in \operatorname{Rco} A \subseteq \operatorname{Rco} E$, thank to Remark 3.3: in fact $\lambda_{2}(\xi)-\lambda_{1}(\xi) \geq \tilde{b}-\tilde{a}=\max _{(a, b) \in K} b-\tilde{a}$, that is $\lambda_{1}(\xi) \leq \tilde{a}$, and $\lambda_{2}(\xi)=\tilde{b}$.

The formula for the interior of Rco $E$ follows from Proposition 2.5 and from the fact that Pco $E=$ Rco $E$, as we have just showed.

\section{THE EXISTENCE THEOREM}

In this section we are going to show Theorem 1.1. The proof will be a direct combination of Theorem 4.5 and of Proposition 4.2. To do this it will be useful to define the so called approximation property (this definition is given in [3]).

Definition 4.1. Let $E \subset K(E) \subset \mathbb{R}^{2 \times 2}$. We say that $E$ and $K(E)$ have the approximation property if there exists a family of closed sets $E_{\delta}$ and $K\left(E_{\delta}\right), \delta>0$, such that

1) $E_{\delta} \subset K\left(E_{\delta}\right) \subset \operatorname{int} K(E)$ for every $\delta>0$;

2) for every $\varepsilon>0$ there exists $\delta_{0}=\delta_{0}(\varepsilon)>0 \operatorname{such}$ that $\operatorname{dist}(\eta, E) \leq \varepsilon$ for every $\eta \in E_{\delta}$ and $\delta \in\left[0, \delta_{0}\right]$;

3) if $\eta \in \operatorname{int} K(E)$ then $\eta \in K\left(E_{\delta}\right)$ for every $\delta>0$ sufficiently small.

We can now show the following result.

Proposition 4.2. Let $E$ be defined by

$$
E=\left\{\xi \in \mathbb{R}^{2 \times 2}:\left(\lambda_{1}(\xi), \lambda_{2}(\xi)\right) \in K\right\}
$$


with $K$ compact satisfying (1.4). Then $E$ and $\mathrm{Rco} E$ have the approximation property with $K\left(E_{\delta}\right)=\mathrm{Rco}_{E_{\delta}}$, if

$$
E_{\delta}=\bigcup_{(a, b) \in K} E_{\delta}^{(a, b)}, \quad E_{\delta}^{(a, b)}=\left\{\xi \in \mathbb{R}^{2 \times 2}:\left(\lambda_{1}(\xi), \lambda_{2}(\xi)\right)=(a-\delta, b-\delta)\right\}
$$

for $0 \leq \delta \leq \min _{(a, b) \in K} a / 2$.

It will be useful the following result due to Cardaliaguet and Tahraoui [1]:

Proposition 4.3. For every $\theta \geq 0$ the function $H_{\theta}: \mathbb{R}^{2 \times 2} \rightarrow \mathbb{R}$ defined by

$$
H_{\theta}(\xi)=\max \left\{\lambda_{1}(\xi) \lambda_{2}(\xi)+\theta\left(\lambda_{2}(\xi)-\lambda_{1}(\xi)\right)-\theta^{2}, 0\right\}
$$

is rank one convex.

Remark 4.4. In [1] Cardaliaguet and Tahraoui show that the function $H_{\theta}$ is polyconvex.

We can now prove Proposition 4.2 .

Proof. We remark that $E_{\delta}$ is compact: this will let us use the representation Theorem 3.1. In the following three steps, we show the three conditions of the approximation property respectively.

1) $\operatorname{Rco}\left(E_{\delta}\right) \subset \operatorname{intRco} E, \forall \delta>0$ :

Let $(a, b) \in K$ be fixed. Then we have

$$
\begin{aligned}
E_{\delta}^{(a, b)} & \subseteq\left\{\xi \in \mathbb{R}^{2 \times 2}: \lambda_{1}(\xi) \lambda_{2}(\xi)<a b, \lambda_{2}(\xi)<b\right\} \\
& =\operatorname{int} \operatorname{Rco}\left\{\xi \in \mathbb{R}^{2 \times 2}:\left(\lambda_{1}(\xi), \lambda_{2}(\xi)\right)=(a, b)\right\} \\
& \subseteq \operatorname{int} \operatorname{Rco} E
\end{aligned}
$$

this implies, if we pass to the union over $K$, that

$$
E_{\delta} \subseteq \text { int } \operatorname{Rco} E
$$

From this inclusion we can infer that

$$
\operatorname{Rco} E_{\delta} \subseteq \operatorname{Rco}(\text { int } \operatorname{Rco} E)=\text { int } \operatorname{Rco} E
$$

as the interior of Rco $E$ is rank one convex. In fact let $\xi, \xi+A \in \operatorname{int} \operatorname{Rco} E$, with $r k(A)=1$, that is, thank to Theorem 3.1, for every $\theta \in\left[0, \max _{(a, b) \in K} b\right]$

$$
\begin{gathered}
\lambda_{1}(\xi) \lambda_{2}(\xi)+\theta\left(\lambda_{2}(\xi)-\lambda_{1}(\xi)\right)<\max _{(a, b) \in K} a b+\theta(b-a) \\
\lambda_{1}(\xi+A) \lambda_{2}(\xi+A)+\theta\left(\lambda_{2}(\xi+A)-\lambda_{1}(\xi+A)\right)<\max _{(a, b) \in K} a b+\theta(b-a) .
\end{gathered}
$$

We want to show that

$$
\xi+s A \in \operatorname{int} \operatorname{Rco} E, s \in[0,1]
$$

Surely $\xi+s A \in \operatorname{Rco} E$, because $\xi, \xi+A \in \operatorname{Rco} E$. Now, let us suppose that there exists $\bar{\theta} \in[0, \underset{(a, b) \in K}{\max } b]$ such that

$$
\max _{(a, b) \in K} a b+\bar{\theta}(b-a)=\lambda_{1}(\xi+s A) \lambda_{2}(\xi+s A)+\bar{\theta}\left(\lambda_{2}(\xi+s A)-\lambda_{1}(\xi+s A)\right) .
$$


We can assume that $\bar{\theta} \neq \max _{(a, b) \in K} b$. In fact, due to $(2.3)$

$$
\left[\max _{(a, b) \in K} b\right]^{2}=\max _{(a, b) \in K}\left\{a b+\left[\max _{(a, b) \in K} b\right](b-a)\right\}
$$

therefore if we choose $\theta=\max _{(a, b) \in K} b$ in (4.1) and in (4.2) we have $\lambda_{2}(\xi), \lambda_{2}(\xi+A)<\max _{(a, b) \in K} b$ and $\lambda_{2}(\xi+s A)=$ $\max _{(a, b) \in K} b$. This is a contradiction as $\lambda_{2}$ is a norm over $\mathbb{R}^{2 \times 2}$. Therefore we can write

$$
\bar{\theta}^{2}<\max _{(a, b) \in K} a b+\bar{\theta}(b-a)=\lambda_{1}(\xi+s A) \lambda_{2}(\xi+s A)+\bar{\theta}\left(\lambda_{2}(\xi+s A)-\lambda_{1}(\xi+s A)\right) .
$$

Using the expression of the function $H_{\bar{\theta}}$ defined in Proposition 4.3, we have

$$
\begin{aligned}
H_{\bar{\theta}}(\xi+s A) & =\lambda_{1}(\xi+s A) \lambda_{2}(\xi+s A)+\bar{\theta}\left(\lambda_{2}(\xi+s A)-\lambda_{1}(\xi+s A)\right)-\bar{\theta}^{2} \\
& =\max _{(a, b) \in K} a b+\bar{\theta}(b-a)-\bar{\theta}^{2}>0 .
\end{aligned}
$$

Thanks to the fact that $H_{\bar{\theta}}(\xi)$ is rank one convex, from Proposition 4.3

$$
0<H_{\bar{\theta}}(\xi+s A) \leq s H_{\bar{\theta}}(\xi+A)+(1-s) H_{\bar{\theta}}(\xi) \leq \max \left\{H_{\bar{\theta}}(\xi), H_{\bar{\theta}}(\xi+A)\right\}
$$

Without loss of generality we can assume that $\max \left\{H_{\bar{\theta}}(\xi), H_{\bar{\theta}}(\xi+A)\right\}=H_{\bar{\theta}}(\xi+A)$. If $H_{\bar{\theta}}(\xi+A)=0$ we have a contradiction. If $H_{\bar{\theta}}(\xi+A)>0$, we have, as $\xi+A \in \operatorname{int} \operatorname{Rco} E$

$$
H_{\bar{\theta}}(\xi+A)=\lambda_{1}(\xi+A) \lambda_{2}(\xi+A)+\bar{\theta}\left(\lambda_{2}(\xi+A)-\lambda_{1}(\xi+A)\right)-\bar{\theta}^{2}<\max _{(a, b) \in K} a b+\bar{\theta}(b-a)-\bar{\theta}^{2}
$$

and so we have obtained

$$
H_{\bar{\theta}}(\xi+s A)=\max _{(a, b) \in K} a b+\bar{\theta}(b-a)-\bar{\theta}^{2} \leq H_{\bar{\theta}}(\xi+A)<\max _{(a, b) \in K} a b+\bar{\theta}(b-a)-\bar{\theta}^{2}
$$

which is a contradiction.

2) $\forall \varepsilon>0 \exists \delta_{0}=\delta_{0}(\varepsilon)>0: \operatorname{dist}(\eta, E) \leq \varepsilon \forall \eta \in E_{\delta}, \delta \in\left[0, \delta_{0}\right]$ :

Let $\eta \in E_{\delta}$; then there exists $(a, b) \in K$ such that $\eta \in E_{\delta}^{(a, b)}$. We define

$$
X=\left(\begin{array}{ll}
a & 0 \\
0 & b
\end{array}\right) \in E
$$

Let $A, B \in \mathcal{O}(2)$ be such that $A \eta B=\left(\begin{array}{cc}\lambda_{1}(\eta) & 0 \\ 0 & \lambda_{2}(\eta)\end{array}\right)$. Then

$$
\left\|\eta-A^{-1} X B^{-1}\right\|=\left\|A \eta B-A A^{-1} X B^{-1} B\right\|=\|A \eta B-X\|=\sqrt{2 \delta^{2}}
$$

This implies that

$$
\operatorname{dist}(\eta, E) \leq\left\|\eta-A^{-1} X B^{-1}\right\|=\sqrt{2 \delta^{2}} \rightarrow 0, \delta \rightarrow 0 ;
$$

moreover this limit is uniform with respect to $\eta$. 
3) If $\eta \in \operatorname{intRco} E$ then $\eta \in \operatorname{Rco} E_{\delta} \forall \delta>0$ sufficiently small:

Let $\eta \in \operatorname{intRco} E$; if $\left(\lambda_{1}(\eta), \lambda_{2}(\eta)\right)=(x, y)$ thanks to Theorem 3.1 we have to show the following implication:

$$
\begin{gathered}
x y+\theta(y-x)<\max _{(a, b) \in K} a b+\theta(b-a) \forall \theta \in\left[0, \max _{(a, b) \in K} b\right] \\
\Downarrow \\
x y+\theta(y-x)<\max _{(a, b) \in K}(a-\delta)(b-\delta)+\theta(b-a)
\end{gathered}
$$

uniformly with respect to $\theta \in\left[0, \max _{(a, b) \in K} b-\delta\right]$. For this it is sufficient to show that

$$
\lim _{\delta \rightarrow 0} \max _{(a, b) \in K}(a-\delta)(b-\delta)+\theta(b-a)=\max _{(a, b) \in K} a b+\theta(b-a)
$$

uniformly with respect to $\theta \in\left[0, \max _{(a, b) \in K} b-\delta\right]$. We have, as $(a-\delta)(b-\delta)+\theta(b-a) \geq 0 \forall \delta \in\left[0, \min _{(a, b) \in K} a / 2\right]$,

$$
\begin{aligned}
\left|\max _{(a, b) \in K}(a-\delta)(b-\delta)+\theta(b-a)-\max _{(a, b) \in K} a b+\theta(b-a)\right| & \leq \max _{(a, b) \in K}|(a-\delta)(b-\delta)+\theta(b-a)-a b-\theta(b-a)| \\
& \leq \max _{(a, b) \in K} \delta(a+b+\delta) \rightarrow 0, \delta \rightarrow 0
\end{aligned}
$$

uniformly with respect to $\theta \in\left[0, \max _{(a, b) \in K} b-\delta\right]$. Consequently we showed the third condition of the approximation property too.

\subsection{Proof of the existence theorem}

We are going to recall an abstract existence theorem (established by Dacorogna and Pisante [4]) that we will apply.

Theorem 4.5. Let $\Omega \subset \mathbb{R}^{2}$ be an open set. Let $E \subset \mathbb{R}^{2 \times 2}$ be a compact set. Assume that $E$ and $\mathrm{Rco} E$ have the approximation property with $K\left(E_{\delta}\right)=\operatorname{Rco} E_{\delta}$. Let $\varphi \in C_{\text {piec }}^{1}\left(\bar{\Omega} ; \mathbb{R}^{2}\right)$ be such that

$$
D \varphi(x) \in E \cup \operatorname{int} \operatorname{Rco} E \text {, a.e. in } \Omega \text {. }
$$

Then there exists $u \in \varphi+W_{0}^{1, \infty}\left(\Omega ; \mathbb{R}^{2}\right)$ such that

$$
D u(x) \in E \text {, a.e. in } \Omega \text {. }
$$

Our theorem then follows immediately. In fact, $E$ is compact and we verified that $E$ and Rco $E$ have the approximation property in Proposition 4.2. Then we obtain the existence Theorem 1.1 thanks to Theorem 4.5.

\section{Representation of Rco $E$}

In this section we are going to give an explicit formula of Rco $E$ for a set $E$ (1.2) defined by a set $K$ composed by a finite number of points. We will treat the cases in which $K$ is composed by one, two elements, and finally a particular $K$ composed by three elements; for the general formula and for its proof we refer to [2]. We recall that the representation of Rco $E$, for $E$ defined by $K$ composed by one element was already obtained by Dacorogna and Marcellini (see [3]); Cardaliaguet and Tahraoui in [1] showed the formula for the case of $K$ composed by two elements.

To give the representation of Rco $E$ it is sufficient to give the formula of the function $\sigma$ defined by (2.1): indeed Rco $E=\left\{\xi \in \mathbb{R}^{2 \times 2}: \lambda_{2}(\xi) \leq \sigma\left(\lambda_{1}(\xi)\right)\right\}$. 
Proposition 5.1. Let $E$ be defined by (1.2) with $K=\{(a, b), 0<a \leq b\}$. Then

$$
\sigma(x)=\inf \left\{\frac{a b}{x}, b\right\} .
$$

Proposition 5.2. Let $E$ be defined by (1.2) with $K=\left\{\left(a_{1}, b_{1}\right),\left(a_{2}, b_{2}\right), 0<a_{i} \leq b_{i}, i=1,2\right\}$. Without loss of generality we can assume that $a_{1} \geq a_{2}$. Then

1) If $a_{1} b_{1}>a_{2} b_{2}$ and $b_{2}>b_{1}$ then

$$
\sigma(x)=\inf \left\{\frac{a_{1} b_{1}}{x}, b_{2}, \frac{\theta(1,2) x+a_{1} b_{1}+\theta(1,2)\left(b_{1}-a_{1}\right)}{x+\theta(1,2)}\right\}
$$

where $\theta(1,2)=\frac{a_{1} b_{1}-a_{2} b_{2}}{b_{2}-a_{2}-\left(b_{1}-a_{1}\right)}$.

2) If $b_{1} \geq b_{2}$ then

3) If $b_{1}<b_{2}$ and $a_{1} b_{1} \leq a_{2} b_{2}$, then

$$
\sigma(x)=\inf \left\{\frac{a_{1} b_{1}}{x}, b_{1}\right\}
$$

$$
\sigma(x)=\inf \left\{\frac{a_{2} b_{2}}{x}, b_{2}\right\} .
$$

Proposition 5.3. Let $E$ be defined by (1.2) with $K=\left\{\left(a_{i}, b_{i}\right), 0<a_{i} \leq b_{i}, i=1,2,3\right\}$. We define

$$
\theta(i, j)=\frac{a_{i} b_{i}-a_{j} b_{j}}{b_{j}-a_{j}-\left(b_{i}-a_{i}\right)} .
$$

Let us suppose that $a_{1} b_{1}>a_{2} b_{2}>a_{3} b_{3}, b_{3}>\max \left\{b_{1}, b_{2}\right\}, b_{2}-a_{2}>b_{1}-a_{1}$ and $\theta(1,2)<\theta(1,3)$. Then

$$
\sigma(x)=\inf \left\{\frac{a_{1} b_{1}}{x}, b_{3}, \frac{x \theta(j, j+1)+a_{j} b_{j}+\theta(j, j+1)\left(b_{j}-a_{j}\right)}{x+\theta(j, j+1)}, j=1,2\right\} .
$$

Acknowledgements. I would like to thank Bernard Dacorogna for having proposed me the subject of this article and for his scientific help; I'm very grateful to the referees for their precious remarks and suggestions. As well, I beneficiated of some discussions with Ana Margarida Fernandes Ribeiro and Giovanni Pisante.

\section{REFERENCES}

[1] P. Cardaliaguet and R. Tahraoui, Equivalence between rank-one convexity and polyconvexity for isotropic sets of $\mathbb{R}^{2 \times 2}$. I. Nonlinear Anal. 50 (2002) 1179-1199.

[2] G. Croce, Ph.D. Thesis (2004).

[3] B. Dacorogna and P. Marcellini, Implicit partial differential equations. Progr. Nonlinear Diff. Equ. Appl. 37 (1999).

[4] B. Dacorogna and G. Pisante, A general existence theorem for differential inclusions in the vector valued case. Submitted.

[5] M. Gromov, Partial differential relations. Ergeb. Math. Grenzgeb. 9 (1986).

[6] R.A. Horn and C.R. Johnson, Topics in matrix analysis. Cambridge University Press, Cambridge (1991).

[7] J. Kolář, Non-compact lamination convex hulls. Ann. Inst. H. Poincaré Anal. Non Linéaire 20 (2003) 391-403.

[8] S. Müller and V. Šverák, Convex integration for Lipschitz mappings and counterexamples to regularity. Ann. Math. 157 (2003) $715-742$.

[9] R.T. Rockafellar, Convex analysis. Princeton Landmarks in Mathematics. Princeton University Press, Princeton, NJ (1997). Reprint of the 1970 original, Princeton Paperbacks. 